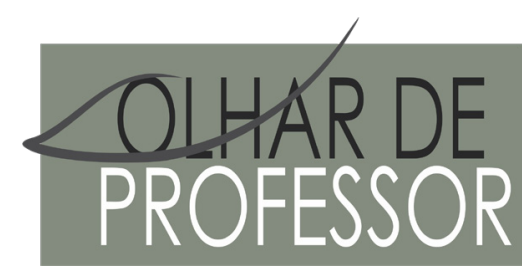

DOI: 10.5212/OLHARPROFR.V.23.2020.15985.209209226496.0614

\title{
COVID-19 Y VIOLENCIA DE GÉNERO E INTRAFAMILIAR: LA ENSEÑANZA DE LA BIOLOGÍA MÁS ALLÁ DE LOS CONTENIDOS ESPERADOS
}

\author{
COVID-19 AND GENDER AND INTRAFAMILY VIOLENCE: THE TEACHING OF BIOLOGY BEYOND THE \\ EXPECTED CONTENTS \\ COVID-19 E VIOLENNCIA DE GÊNERO E INTRAFAMLLIAR: O ENSINO DE BIOLOGIA PARA ALÉM DOS \\ CONTEÚDOS ESPERADOS
}

YONIER ALEXANDER OROZCO MARÍN* LADY JOHANNA HERRERA VARGAS**

\begin{abstract}
Resumen: El trabajo presenta el desarrollo de una secuencia didáctica que tuvo como objetivo promover reflexiones por parte de estudiantes del grado sexto (entre 10 y 12 años) de una Institución Educativa privada de la ciudad de Bogotá Colombia, desde las clases de biología, sobre las dinámicas sociales asociadas al incremento de la violencia de género e intrafamiliar durante el periodo de cuarentena, por causa del COVID-19. La actividad consistió en el desarrollo de un taller, partiendo de la realización de lecturas de textos que abordan el incremento de la violencia intrafamiliar y de género en Bogotá en este tiempo de pandemia. Encontramos que el estudiantado destaca el aumento del trabajo doméstico para las mujeres en su hogar, que algunos integrantes de sus familias han pasado por episodios de violencia de género o intrafamiliar, discutiendo algunas posibilidades para superar esa violencia. Defendemos la importancia de la enseñanza de la biología para problematizar asuntos relacionados a la justicia social, principalmente en tiempos de COVID-19.
\end{abstract}

Palabras-clave: Educación para la justicia social. Educación y género. Enseñanza de la biología. Violencia intrafamiliar. violencia de género.

Resumo: O trabalho apresenta o desenvolvimento de uma proposta de ensino que teve como objetivo promover reflexões de alunas e alunos da sexta série (entre 10 e 12 anos) de uma instituição de ensino particular da cidade de Bogotá, Colômbia, nas aulas de biologia, sobre a dinâmica de aumento da violência de gênero e intrafamiliar durante o período de quarentena, devido á pandemia do COVID-19. A atividade consistiu em desenvolver uma oficina baseada na leitura de textos que abordam o aumento da violência doméstica e de gênero em Bogotá durante o período de pandemia. Constatamos que os estudantes destacam o aumento do trabalho doméstico para as mulheres nas suas casas, que alguns membros de suas famílias já passaram por episódios de violência de gênero ou intrafamiliar, discutindo algumas possibilidades de superação dessa violência. Defendemos a importância do ensino de biologia para problematizar questões relacionadas à justiça social, principalmente nos tempos do COVID-19.

Palavras-chave: Educação para a justiça social. Educação e gênero. Ensino da biologia. Violência intrafamiliar. Violência de gênero.

\footnotetext{
* Estudiante del Doctorado en Educación Científica y Tecnológica, Universidade Federal de Santa Catarina. Bolsista CAPES. E-mail: apmusicomano@gmail.com

${ }^{* *}$ Magíster en desarrollo sustentable y gestión ambiental de la universidad Distrital Francisco José de Caldas. Docente Investigadora de la División de Investigación DITA en la Corporación Tecnológica Industrial Colombiana. E-mail: lajoheva@gmail. com
} 


\begin{abstract}
The work presents the development of a didactic sequence that aimed to promote reflections by sixthgrade students (between 10 and 12 years old) from a private educational institution in the city of Bogotá-Colombia, from biology classes, on the dynamics activities associated with the increase in gender and intra-family violence during the quarantine period, due to COVID-19. The activity consisted of developing a workshop based on the reading of texts that address the increase in domestic and gender violence in Bogotá during this time of the pandemic. We found that the students highlight the increase in domestic work for women in their home, that members of their families have gone through episodes of gender or intra-family violence, discussing some possibilities to overcome this violence. We defend the importance of teaching biology to problematize issues related to social justice, mainly in the days of COVID-19.
\end{abstract}

Keywords: Biology education. Domestic violence. Education and gender. Education for social justice. Gender violence.

\title{
INTRODUCCIÓN
}

En un mundo convulsionado como el actual, en el que se visibilizan las brechas de desigualdad, adquiere mayor relevancia la educación como derecho fundamental y social, el cual debe ser garantizado para todos, ya que, la educación busca la formación integral de las personas, a partir del desarrollo de conocimientos, habilidades y valores (MINISTERIO DE EDUCACIÓN, 2016).

En cuanto al desarrollo del conocimiento en Colombia se elaboraron los derechos básicos de aprendizaje (DBA) y los Estándares Básicos de Competencias (EBC), los cuales parten de la premisa que el aprendizaje debe brindar un contexto cultural e histórico a quien aprende. De tal forma que los DBA establecen los conocimientos estructurantes que se deben abordar en cada grado y área particular y los EBC buscan transformar la visión simplista de la educación de la transmisión y memorización de contenidos (MINISTERIO DE EDUCACIÓN, 2016; SUBDIRECCIÓN DE REFERENTES Y EVALUACIÓN DE LA CALIDAD EDUCATIVA DIRECCIÓN DE CALIDAD, 2013, p.20).

Sin embargo, pese a que los DBA y los EBC buscan ir más allá de los contenidos, en la práctica, generalmente estos prevalecen en las áreas en donde el conocimiento se imparte de una forma fragmentada, sin un contexto cultural o histórico. Desconocer el carácter socio-histórico-cultural de la educación y la necesidad de expresar en esta las costumbres y el momento en la vida de un pueblo, es ignorar que los procesos de enseñanza-aprendizaje están sujetos a las circunstancias en que se expresan, de aquí lo nocivo de copiar teorías, métodos y procedimientos que resultan ajenos a la cultura, la historia y la realidad concreta de un pueblo (RODRÍGUEZ GONZÁLEZ; PETEIRO SANTAYA; RODRÍGUEZ WONG, 2007).

Así pues, es necesario reflexionar sobre el papel del docente de biología en la coyuntura social causada por el COVID-19, la cual ha generado diferentes retos sociales y ha obligado a desarrollar nuevas estrategias en los procesos de enseñanza-aprendizaje por parte de docentes y estudiantes, lo que, a su vez ha mostrado problemáticas sociales como la violencia intrafamiliar, la violencia de género, las brechas de desigualdad, entre otras.

Por ejemplo, en Colombia en medio de la cuarentena "se reportó un aumento de 170 por ciento en las llamadas que reportaron casos de violencia intrafamiliar. Y se han presentado 12 casos de feminicidio”(CONSEJERÍA PRESIDENCIAL PARA LA EQUIDAD DE LA MUJER., 2020, p.16)

Ante esta realidad y sí se busca que los conocimientos, habilidades y valores de los estudiantes estén enmarcados en un contexto socio-histórico-cultural, es relevante preguntarse ¿cómo puede abordarse la violencia de género e intrafamiliar desde la clase de biología, puesto que este no es un tema que se encuentra en el currículo? Principalmente, en estos tiempos en los cuales parece reforzarse el imaginario de que la enseñanza de la biología debería privilegiar los asuntos epidemiológicos y/o científicos sobre el COVID-19 únicamente.

En consecuencia, el presente artículo aborda la experiencia de un ejercicio hecho en el aula con 50 estudiantes del grado sexto, con edades entre los 10 y los 12 años, de un colegio privado de la ciudad de Bogotá, Colombia. El objetivo de la experiencia didáctica fue promover la construcción de reflexiones por parte de las y los estudiantes, desde las clases de biología, sobre las dinámicas sociales asociadas al 
incremento de la violencia de género e intrafamiliar durante el periodo de cuarentena, por causa de un agente biológico como el COVID-19.

\section{METODOLOGÍA}

La experiencia que aquí se aborda se realizó en medio de la cuarentena del COVID-19 desde la virtualidad. Se elaboró un taller para desarrollar por los 50 estudiantes de grado sexto y sus familias, en el mes de abril de 2020. El taller fue explicado al estudiantado en una sesión de 90 minutos, y después, este mismo era resuelto de manera asincrónica en casa. El taller consistió en las siguientes actividades divididas en dos partes:

\section{PRIMERA PARTE: CONTEXTUALIZACIÓN}

La actividad consistió en leer dos textos, con algún familiar. A continuación, los textos:

Texto 1: El coronavirus golpea tres veces a las mujeres: por la salud, por la violencia doméstica y por cuidar de los otros. Fuente: https://news.un.org/es/story/2020/03/1471872

Texto 2: Que la violencia intrafamiliar no sea parte de esta cuarentena por coronavirus. Fuente: https://bogota.gov.co/mi-ciudad/integracion-social/violencia-intrafamiliar-cuarentena-coronavirus

\section{SEGUNDA PARTE: CUESTIONARIO}

El estudiantado debería responder nueve preguntas, sin embargo, por limitaciones de espacio abordamos tres de estas, siendo:

a) Según el texto, en la mayoría de hogares el periodo de la cuarentena hará que a muchas mujeres se les recargue más trabajo doméstico. ¿Por qué ocurre esta situación? ¿Esto también ocurre en tu hogar? Explica tus respuestas.

b) Completa una tabla haciendo una encuesta a todas las personas que viven en tu casa (Incluyéndote) en este periodo de cuarentena con las siguientes preguntas: ¿ Alguna vez ha sufrido o ha presenciado algún caso de violencia de género o infantil? (Responder SI o NO) y describe la situación.

c) ¿Cómo puedes ayudar en tu día a día para evitar que en tu hogar haya violencia intrafamiliar, de género o infantil? Menciona por lo menos tres ejemplos y dibújalos.

\section{RESULTADOS Y DISCUSIÓN}

Al cuestionar al estudiantado sobre si el recargo de más trabajo doméstico a las mujeres también ocurría en su hogar, cerca de una cuarta parte manifestó que sí (Figura 1).

FIGURA 1. PORCENTAJE DE ESTUDIANTES QUE MANIFESTÓ QUE EN SUS HOGARES HA AUMENTADO EL RECARGO DE TRABAJO DOMÉSTICO A LAS MUJERES [71\%] Y QUIENES NO [29\%] [N=50).

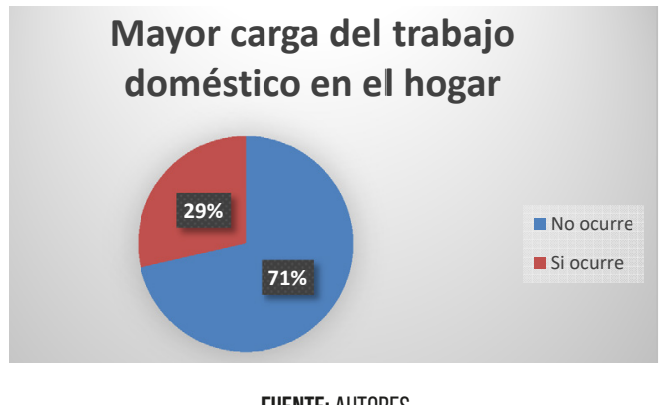

FUENTE: AUTORES 
La figura 2 muestra los resultados que obtuvo el estudiantado en sus hogares al preguntar: ¿Alguna vez ha sufrido o ha presenciado algún caso de violencia de género o infantil? a cada miembro de la familia.

FIGURA 2. ENCUESTA FAMILIAR ¿ALGUNA VEZ HA SUFRIDO O HA PRESENCIADO ALGÚN CASO DE VIOLENCIA DE GÉNERO O INFANTIL?

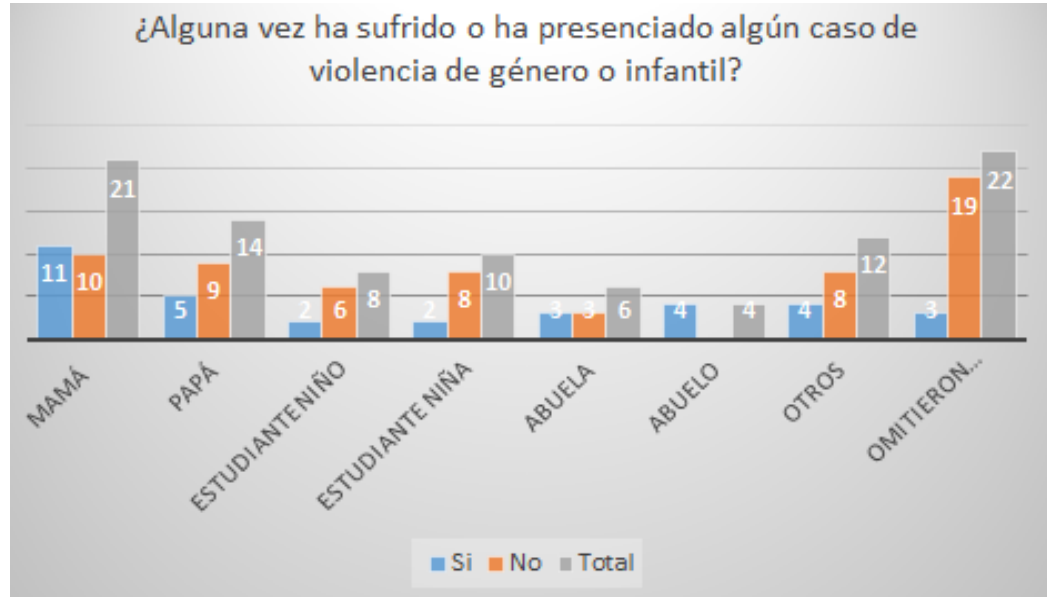

FUENTE: AUTORES

Sobre la pregunta ¿Cómo puedes ayudar en tu día a día para evitar que en tu hogar haya violencia intrafamiliar, de género o infantil? hubo una gran diversidad de respuestas, sin embargo, en la figura 3 se observan las respuestas más comunes.

FIGURA 3. ¿CÓMO PUEDES AYUDAR EN TU DÍA A DÍA PARA EVITAR QUE EN TU HOGAR HAYA VIOLENCIA INTRAFAMILIAR, DE GÉNERO O INFANTIL?

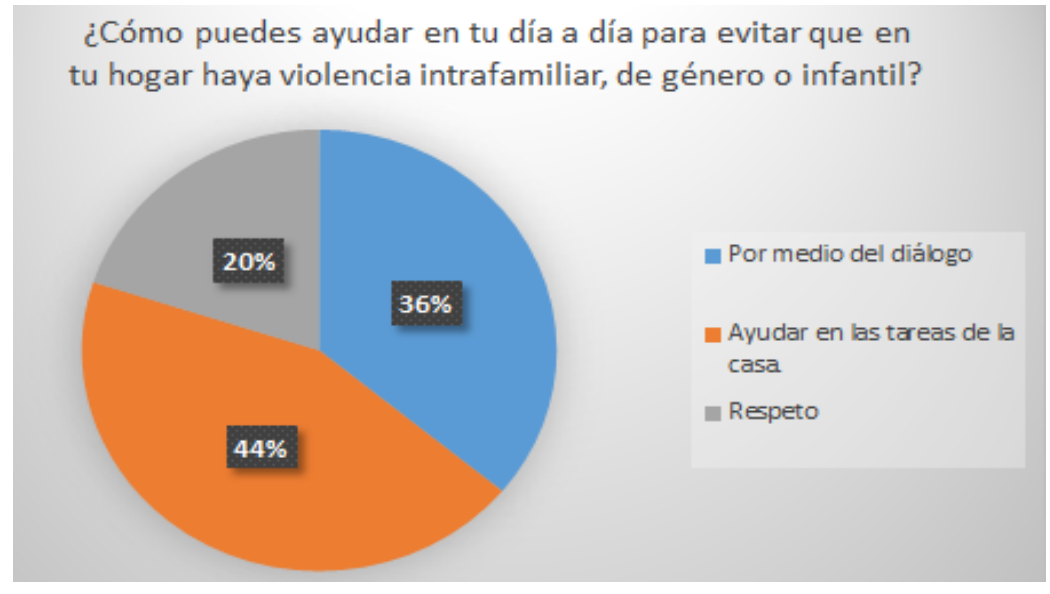

FUENTE: AUTORES

Los resultados colocados demuestran que al sugerir que la enseñanza de la biología se debe limitar a abordar los aspectos científicos y epidemiológicos sobre el COVID-19, estaríamos perdiendo el potencial de abordar problemas también urgentes, de carácter más social, histórico y cultural que salen a la luz y se agravan durante la pandemia. El COVID-19 no es únicamente un agente biológico que puede afectar nuestros cuerpos, sino que también, un agente que profundiza las desigualdades sociales que Colombia ya presentaba, especialmente la violencia de género e intrafamiliar. Consideramos entonces que este momento también llama a la enseñanza de la biología a resignificarse pensando en esas realidades.

En la dimensión social, como lo menciona Herrera (2020) el COVID-19 abrió las puertas (o mejor, las cerró) para el aumento de las grietas de inequidad en un sistema que se pretende paritario, los llamados de alarma que están señalando un repunte en las diversas formas de violencia doméstica e intrafamiliar, ejercidas, como en todos los ámbitos, fundamentalmente por hombres, siendo las víctimas mujeres, niñas, niños, adultos y adultas mayores. Sin embargo, al ser esta violencia naturalizada en la sociedad 
colombiana, pocos son los espacios públicos y privados disponibles para realizar estas discusiones, explicitar y reflexionar sobre cómo superarla. Por esto, la importancia de que en clases de biología, y a pesar de que no sea una propuesta curricular del país, se introduzca esta problematización.

Ballesteros (2020) destaca que las prácticas educativas no pueden seguir fingiendo una normalidad ante la realidad social que ahora se nos presenta, y seguir reproduciendo la competitividad, o la formación de individuos para ver cuál está mejor capacitado que el otro. Estos tiempos nos llaman a repensar la función productivista de individuos capacitados al servicio del capital y del patriarcado, para pensar prácticas de empatía por la tierra y los demás. Empatía y mayor cuidado con nuestro cuerpo y su cuidado, ejemplificado en la mención de un estudiante: “Como niños debemos cuidar nuestro cuerpo, no dejarnos tocar de ninguna persona y no callar ningún acto de violencia intrafamiliar” (sic. estudiante sexto grado,2020).

En ese sentido, es necesario recordar que la biología es la ciencia que estudia “lo vivo”. Enseñarla sin articularla con problemáticas sociales que afectan la vida de determinados grupos sociales sería separarla de sus objetivos intrínsecos. Según lo expresa el mismo estudiantado (Figura 4) es menester problematizar y frenar la violencia de género, ahora más que nunca.

FIGURA 4. DIBUJOS Y REFLEXIONES DEL ESTUDIANTADO, DENUNCIANDO LA NECESIDAD DE ABORDAR PÚBLICAMENTE LA VIOLENCIA DE GÉNERO Y FRENAR ESTE PROBLEMA.

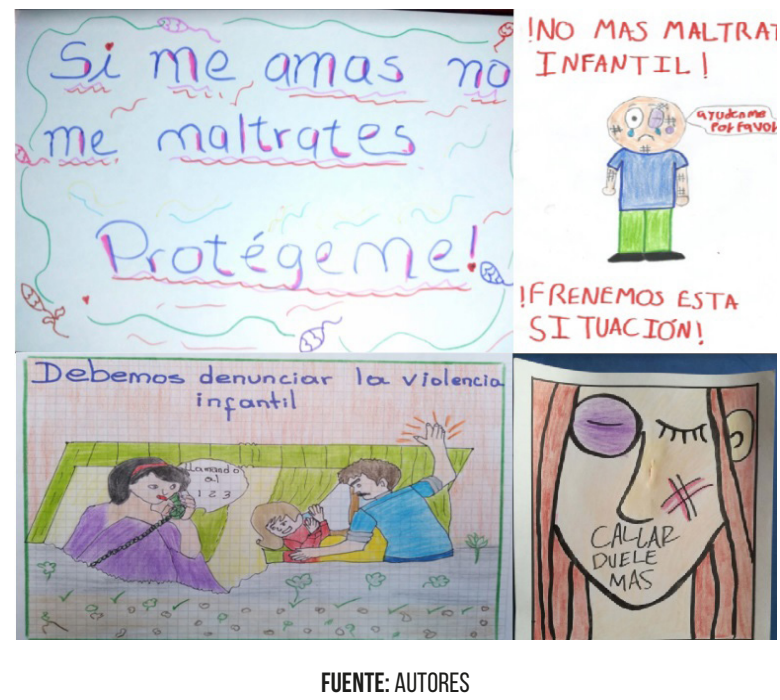

Desde la dimensión cultural, podemos mencionar que este abordaje no es fácil. En el contexto colombiano circula el dicho "los trapos sucios se lavan en casa” haciendo referencia a que problemas dentro de la familia no deben salir al público. Son asuntos que se refugian en lo privado para no ser discutido. Por lo tanto, la escuela como espacio público no acostumbra a ser llamada a tocar estos asuntos, en "respeto" a la privacidad de ese asunto. Una educación realmente pensada en función de la justicia social debe abordar explícitamente estos asuntos, de lo contrario, sería aliada de la perpetuación de esta violencia.

Reconocemos también que este elemento de la privacidad de la violencia de género e intrafamiliar puede haber interferido en las respuestas del estudiantado. Porque al estar en casa no pueden expresar libremente sus respuestas, pues en muchos casos están en presencia del agresor y/o víctimas, que pueden filtrar la información relatada por las y los estudiantes, para que esta no pase al espacio público de la escuela. Ocurrió en el caso de un estudiante, quien en las respuestas de este taller manifestó no existir problemas de violencia intrafamiliar en su casa o por ninguno de sus familiares. Sin embargo, en otra actividad, la cual consistía en una evaluación que debían resolver sincrónicamente e individualmente, con cámara encendida para garantizar la no ayuda de sus acudientes, el estudiante mencionó en una pregunta sobre el aumento de violencia de género en tiempos de pandemia: "Bueno, la violencia sí ha aumentado bastante y pues es por el encierro. Yo en tiempo del Coronavirus he visto mi papa peleando mucho más con mi mama y ahora que mis papas se acaban de separar, a parte de estar triste, he visto que la violencia aumentó y no es bueno, estamos muy estresados todos con la pandemia” (sic. estudiante sexto grado,2020). 
La dimensión cultural está íntimamente relacionada con la dimensión histórica, lo que afirma el estudiante, es la realidad de muchos hogares en el mundo, en medio del momento histórico que estamos viviendo de la pandemia los divorcios han aumentado, la violencia intrafamiliar y de género también han aumentado. Las mujeres se encuentran en un momento en el que los roles tradicionales de género son un mecanismo más de vulnerabilidad y de violencia de género, puesto que, "han visto incrementada su carga de trabajo no remunerado, lo que tiene un efecto directo sobre sus condiciones físicas, económicas y sicológicas” (CONSEJERÍA PRESIDENCIAL PARA LA EQUIDAD DE LA MUJER., 2020, p.6)

Un ejemplo de ello se ve reflejado en la respuesta de dos estudiantes frente a la pregunta de la sobrecarga doméstica en el hogar, la primera estudiante afirmó que "Si ya que yo estoy estudiando y eso implica mas trabajo en las cosas del hogar para mi mamá y en el caso de mi abuela debe cuidar a mi bisabuela ya que es un adulto mayor de 90 años.” (sic. estudiante sexto grado,2020). Mientras que el segundo estudiante afirmó: "Si por que la persona encargada en mi hogar es mi abuelita,le toca estar pendiente de las comidas de mas personas al día en la casa ella esta pendiente de nuestras onces por que nosotros nos encontramos en los computadores y cuadernos"(sic. estudiante sexto grado, 2020). Estos dos testimonios reflejan los roles tradicionales de género como un elemento transgresor para las mujeres.

Por otro lado, hay algo que destacar de la dimensión histórica y es el reconocimiento de que las violencias de género e intrafamiliar no son algo nuevo, es algo que viene sucediendo en el espacio privado de los hogares, lo que se vio reflejado en las respuestas de las abuelas, donde una de ellas afirmó que "antes se veía mucho - un claro ejemplo el machismo" (sic, abuela estudiante grado sexto, 2020).

Sin embargo, llama la atención que los abuelos negaron haber vivido alguna situación de violencia intrafamiliar o de género, esta respuesta puede darse por mantener la privacidad del hogar o porque realmente ellos no reconocen las acciones presentes o pasadas como violencia, sino que son parte de su cultura o costumbres donde los roles de género tradicionales se llevan a cabo sin discusión.

Además, estos roles, usualmente generan una división del espacio, donde el lugar de las mujeres es lo privado, es decir la casa, la cocina, el cuidado, mientras que el lugar de los hombres es lo público, es decir la calle u otros espacios como los laborales.

Finalmente, es de destacar que los roles de género deben ser abordados desde la clase de biología, ya que, es urgente una resignificación de lo que implica ser mujer o ser hombre, en un mundo que sin duda no será el mismo una vez superemos la pandemia.

\section{CONSIDERACIONES FINALES}

Con este trabajo evidenciamos la necesidad y urgencia de repensar la enseñanza de la biología en estos tiempos de pandemia, con lo cual no pretendemos afirmar que este aspecto se deba limitar a estos tiempos. Destacamos que no se puede continuar “fingiendo" normalidad con la enseñanza de conceptos en una perspectiva de capacitación individual y necesitamos movilizar discusiones más empáticas con relación a la violencia y desigualdad social que en este tiempo se agravian a causa de las nuevas dinámicas impuestas por el agente biológico, el COVID-19.

La violencia de género e intrafamiliar es de esos asuntos que deberían ser abordados de manera pública y permanente en los contextos escolares, particularmente desde la enseñanza de la biología. Con esta experiencia evidenciamos que, a pesar de las dificultades de tratar estos temas con el estudiantado en sus casas y bajo una mayor vigilancia de la familia, entre quienes se pueden encontrar agresores o víctimas intimidadas. Parte del estudiantado destaca el aumento del trabajo doméstico para las mujeres en su hogar, además que algunos integrantes de sus familias (incluyendo madre, padre, abuelas y ellos mismos) han pasado por episodios de violencia de género o intrafamiliar. Sin embargo, es necesario reconocer que en algunos casos se prefirió omitir la respuesta a estas preguntas o afirmar que esta violencia no hace parte de sus hogares, a pesar de que en otros momentos del proceso educativo lleguen a manifestar lo contrario. Con el reconocimiento de estas violencias, fue posible discutir con el estudiantado algunas posibilidades para superar esa violencia, dentro de las cuales se destaca el respeto, el diálogo y una mejor distribución de las tareas domésticas. 
Defendemos que la enseñanza de la biología debe transformar sus prácticas en este periodo de cuarentena, al abordar asuntos que dialoguen de manera más directa con las violencias y desigualdades agudizadas en este periodo de cuarentena.

\section{REFERÊNCIAS}

BALLESTEROS, G. Igualdad educativa y postpandemia. In: PALAU, J. Educación y Pandemia: Una visión académica. UNAM: México, 2020. p. 183-194.

CONSEJERÍA PRESIDENCIAL PARA LA EQUIDAD DE LA MUJER. COVID-19 : Impacto diferencial. Bogotá: [s.n.].

EL CORONAVIRUS GOLPEA TRES VECES A LA MUJERES: por la salud, por la violencia doméstica y por cuidar de los otros. Notícias ONU. 27 de março de 2020. Seção mulher, disponível em: https:// news.un.org/es/story/2020/03/1471872

ESTUPIÑÁN, K. Que la violencia intrafamiliar no sea parte de esta cuarentena. Bogotá, Bogotá D.C. 27 de março de 2020. Integração social. Disponível em: https://bogota.gov.co/mi-ciudad/integracionsocial/violencia-intrafamiliar-cuarentena-coronavirus

HERRERA, M. Géneros, Equidad y Violencia en tiempos de COVID-19: Viejos problemas, nuevos problemas. In: PALAU, J. Educación y Pandemia: Una visión académica. UNAM: México, 2020. p. 174-182.

MINISTERIO DE EDUCACIÓN DE COLOMBIA. Derechos Básicos de Aprendizaje. 1. ed. Panamericana Formas E Impresos S.A.: Bogotá, v. 1, 2016.

RODRÍGUEZ, R.; PETEIRO, L. M.; RODRÍGUEZ, M. T. La educación desde un enfoque histórico social: importancia para el desarrollo humano. Revista Psicología Científica, p. 1-5, 2007.

SUBDIRECCIÓN DE REFERENTESY EVALUACIÓN DE LACALIDADEDUCATIVADIRECCIÓN DE CALIDAD. Implementación de los estándares básicos de competencias en el aula. Bogotá: [s.n.]. Disponível em: <https://www.atlantico.gov.co/images/stories/adjuntos/educacion/eje6at.pdf>. Acesso em: 12 jun. 2020. 